\title{
东江惠州河流段人工基质附着硅藻群落的季节性动态
}

\author{
陈 向,刘 静,何 琦, 林秋奇, 韩博平, 韦桂峰**
}

（暨南大学水生生物研究中心,广州 510632)

\begin{abstract}
摘 要: 硅藻群落结构特征是反映河流水生态状态的重要生物指标, 但我国多数河流中还缺少硅藻种类组成与季节变化 的基本数据. 2010 年用瓷砖片作为人工基质研究东江惠州河流段附着硅藻群落结构的季节性变化,共鉴定出附着硅藻 40 属 153 种(包括亚种和变种), 主要的优势属有曲壳藻属 (Achnanthidium)、异极藻属 (Gomphonema)、直链藻属 (Melosira)、 舟形藻属 (Navicula)、菱形藻属 (Nitzschia)、脆杆藻属 (Synedra) 等. Gomphonema parvulum 和 Nitzschia palea 为该河流段的广 泛性藻种, 且相对丰度的季节性变化明显. $\mathrm{RDA}$ 分析表明, 水温、DO、 $\mathrm{NH}_{4}^{+}-\mathrm{N} 、 \mathrm{PO}_{4}^{3-}-\mathrm{P} 、$ 电导率、 $\mathrm{pH}$ 值等对附着硅藻群落结 构有重要影响. 在丰水期, 水量对于运动能力较强的 N. palea 的相对丰度具有明显的影响.
\end{abstract}

关键词: 附着硅藻;季节性变化;人工底质;东江

\section{Seasonal variations of periphytic diatom community in artificial substrata in Huizhou sec- tion of the Dongjiang River, Guangdong Province, Southern China}

CHEN Xiang, LIU Jing, HE Qi, LIN Qiuqi, HAN Boping \& WEI Guifeng

(Research Center of Hydrobiology, Jinan University, Guangzhou 510632, P. R. China)

Abstract: Although diatom has been widely used in biological or ecological monitoring of rivers, there is little information on diatom communities in most of Chinese rivers. In this study, we reported periphytic diatoms on artificial substrata( tile) in the Huizhou section of the Dongjiang River. We analyzed seasonal variations of the species composition and the relationship between the relative abundance and seasonal environmental factors at five sampling stations in 2010. A total of 153 periphytic diatom species, belonging to 40 genera, were identified. Dominant genera included Achnanthidium, Gomphonema, Melosira, Navicula, Nitzschia and Synedra. Gomphonema parvulum and Nitzschia palea were the common species in all the sampling sites with relative abundance $>1 \%$. However, the relative abundance of the two species varied largely in seasons. Chl. a concentrations of the periphytic algae were positively correlated with the temperature. Redundancy analysis showed that water temperature, $\mathrm{DO}, \mathrm{NH}_{4}^{+}-\mathrm{N}, \mathrm{PO}_{4}^{3-}-\mathrm{P}$, conductivity and $\mathrm{pH}$ were the major factors influencing the community structure of periphytic diatoms. Furthermore, hydrographic condition was crucial to some mobile species such as Nitzschia palea, whose relative abundance was low in summer mainly due to flushing in flood season.

Keywords: Periphytic diatom; seasonal variation; artificial substrata; Dongjiang River

底栖藻类的主要栖息地为溪流的底部、河流沿岸的石头、大型植物及苔藓等底质 ${ }^{[1]}$, 是河流生态系统中 主要的初级生产者之一 ${ }^{[2]}$,其中主要包括蓝藻、绿藻、硅藻和金藻等. 而硅藻是河流及溪流生态系统中物种 多样性最为丰富的群落之一 ${ }^{[3]}$. 相对底栖动物和鱼类,底栖硅藻有很高的繁殖率和很短的生命周期, 能够对 周围的理化因子及生态环境变化进行快速响应,基于这些特征, 硅藻已被广泛用于河流及湖泊的水质生物 监测和生态完整性评价 ${ }^{[4]}$.

硅藻生长环境宽泛, 适应能力强, 从寒冷地区到温泉等环境中, 都有一些相应的硅藻能够正常生长和繁 殖. 不同种类的硅藻有其最适生长的温度范围, 部分种类的适温范围较广 ${ }^{[5]}$. 比如, Gomphonema gracilis 是一 个世界性分布的广泛种, Krammer 等发现此种硅藻在热带及北欧地区广泛存在 ${ }^{[6]}$, 而 Pienitz 等在加拿大的

* 国家水体污染控制与治理科技重大专项项目 (2009ZX07211-009-1) 资助. 2011-12-19 收稿;2012-03-15 收修改 稿. 陈向,男,1984 年生,硕士研究生;E-mail:xiang8234@ 126. com.

** 通信作者;E-mail:tweigf@ jnu. edu.cn. 
研究发现该藻在低温环境中生长良好 ${ }^{[7]}$. 在不同的理化条件下, 河流底栖硅藻组成有较大的差异. 例如, Rothfritz 等在研究植物和石头上的附着硅藻时, 发现各采样点之间的硅藻群落比不同附着介质之间的硅藻 群落变化要大得多 ${ }^{[8]}$. 这种群落组成上有规律的变化成为利用硅藻进行生物或生态监测的理论基础.

对于大型河流而言, 由于底栖硅藻采样比较困难及硅藻附着介质具有空间异质性,难以进行令人满意 的定量分析 ${ }^{[9]}$. 由于人工底质能够提供一致的附着条件, 许多研究人员倾向于选择用人工底质挂片的方法 采集底栖硅藻来对水体进行生态或生物监测. 人工底质具有在面积和附生藻类生长时间可控制性等优 点 ${ }^{[10]}$, 减少重复取样及生境差异所产生的误差, 有利于不同样点之间的比较,也便于进行统计分析 ${ }^{[11]}$, 为底 栖附生硅藻的研究提供了有效的方法. 在过去几十年中, 研究者尝试采用不同种类的底质. Cattaneo 等统计 了 1975-1990 年期间 20 个期刊中用于附着硅藻研究的各种底质的文献,结果表明瓷砖片是采用最为广泛 的人工底质 ${ }^{[9]}$; Tuchman 等比较了以瓷砖片和石头做人工底质的溪流附生硅㮫群落结构, 发现瓷砖片与自然 底质上的硅藻群落更接近 ${ }^{[12]}$. 随着欧洲水框架和美国清洁水计划的执行, 地处温带地区的欧美等发达国家 已建立起相对完整的硅藻生物监测与评估体系 ${ }^{[13]}$. 与欧美国家相比, 热带及亚热带地区发展中国家的硅藻 生物监测则还处于起步阶段 ${ }^{[14]}$, 种类分类系统不成熟, 基础数据缺乏, 同时相应的研究方法也不完善 ${ }^{[15]}$.

我国的多数河流缺少硅藻群落的基础信息, 还难以建立有效的生物监测与评价方法. 本文用瓷砖片作 为人工底质, 调查我国南亚热带典型河流——东江的惠州段底栖硅藻群落的种类组成及其季节性变化, 为 今后利用底栖硅藻进行生物监测提供基础数据.

\section{1 材料与方法}

\section{1 河流概况及采样点的设置}

东江为珠江三大水系之一,干流总长 $523 \mathrm{~km}$, 流经河源、东莞、惠州等重要城市. 惠州河流段是东江的下 游河段, 建有剑潭大坝, 大坝出水经过太园原站 (东深供水工程的取水口) 后进人珠江口.

2010 年对东江惠州河流段底栖附生硅藻进行调查,研究区域主要在惠州市内. 由大坝往上游方向, 依次 共设 5 个采样点, 分别为剑潭大坝 $\mathrm{S} 1\left(23^{\circ} 09^{\prime} \mathrm{N}, 114^{\circ} 19^{\prime} \mathrm{E}\right)$ 、水文站 $\mathrm{S} 2\left(23^{\circ} 06^{\prime} \mathrm{N}, 114^{\circ} 24^{\prime} \mathrm{E}\right)$ 、老水厂 $\mathrm{S} 3$ $\left(23^{\circ} 04^{\prime} \mathrm{N}, 114^{\circ} 25^{\prime} \mathrm{E}\right)$ 、虾村 $\mathrm{S} 4\left(23^{\circ} 08^{\prime} \mathrm{N}, 114^{\circ} 26^{\prime} \mathrm{E}\right)$ 和汝湖 $\mathrm{S} 5\left(23^{\circ} 10^{\prime} \mathrm{N}, 114^{\circ} 27^{\prime} \mathrm{E}\right)$, 其中 S3 点位于西枝江支 流, 与东江干流和支流的交汇口较近, 其他 4 个点都在东江干流中 (图 1). 采样频率为每季度一次, 具体时间 分别为 2010 年的 1 月 (冬季)、4 月(春季)、8 月(夏季)、11 月(秋季).

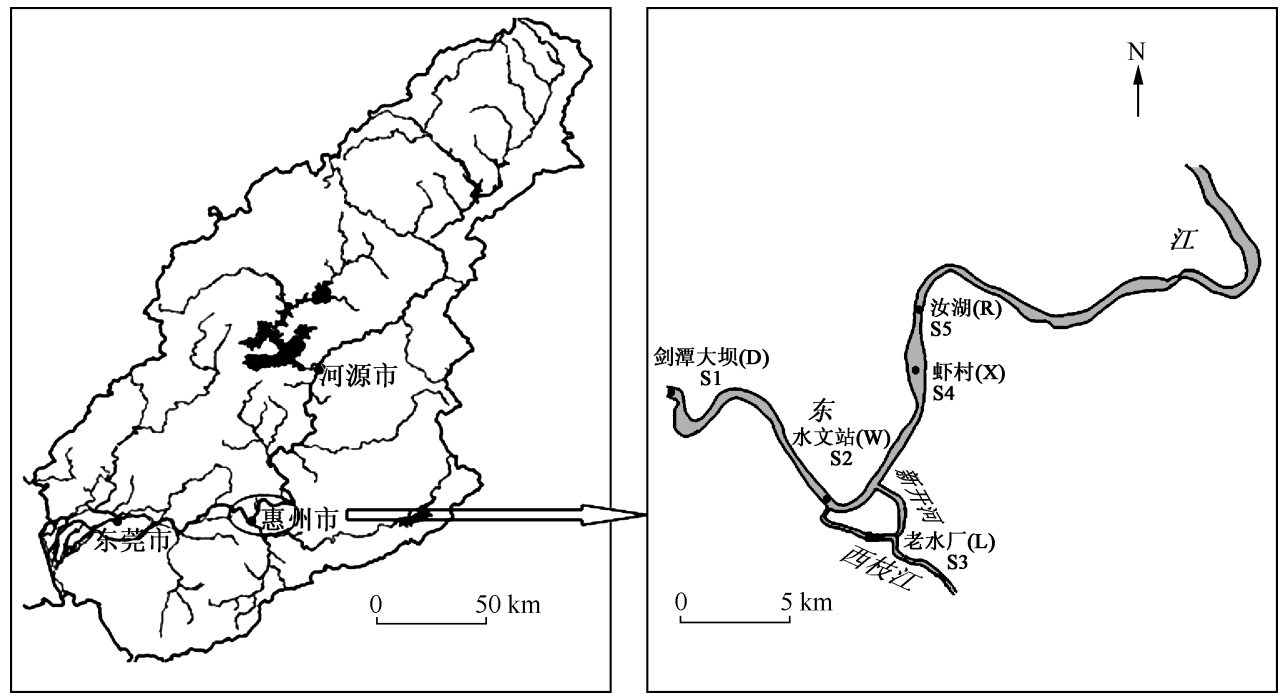

图 1 东江惠州段采样点的分布

Fig. 1 Location of sampling sites in Huizhou section of the Dongjiang River 


\section{2 样品采集与处理}

本实验用规格为 $20 \mathrm{~cm} \times 20 \mathrm{~cm}$ 的瓷砖片作为人工底质研究东江惠州段底栖附生硅藻. 将瓷砖片悬挂在 离河面 $30 \sim 50 \mathrm{~cm}$ 处. 挂片时, 用 YSI-85 型水质仪现场测定水体温度 (WT)、pH 值、溶解氧 ( DO) 和百分溶氧 量 (DO \% ) 、电导率 (Cond) 等理化指标; 透明度 $(\mathrm{SD})$ 用萨氏盘测定. 并用棕色瓶在原处采 $1 \mathrm{~L}$ 水带回实验室, 当天用国标法完成总氮 $(\mathrm{TN})$ 、硝氮 $\left(\mathrm{NO}_{3}^{-}-\mathrm{N}\right)$ 、铵氮 $\left(\mathrm{NH}_{4}^{+}-\mathrm{N}\right)$ 、总磷 $(\mathrm{TP})$ 及正磷酸盐 $\left(\mathrm{PO}_{4}^{3-}-\mathrm{P}\right)$ 等各水化指标 的测定.

参考 Biggs 的挂片时间 ${ }^{[16]}$,2 周后用硬毛牙刷将瓷砖片上的底栖藻类在盘子里刷下,并用水冲洗干净, 将刷取的样品倒人 $100 \mathrm{ml}$ 透明瓶中, 回到实验室后立即抽取 $10 \mathrm{ml}$ 的样品用孔径为 $0.45 \mu \mathrm{m}$ 的微孔滤膜过 滤, 用反复冻融一浸提法测定底栖藻类叶绿素 $\mathrm{a}(\mathrm{Chl} . \mathrm{a})^{[17]}$; 剩下的样品按 $4 \%$ 的比例加甲醛溶液固定, 用于 底栖硅藻的种类鉴定和定量分析. 根据 Battarbee 等提供的方法对样品进行前期处理 ${ }^{[18]}$, 并用折射率为 1.704 的 Naphrax 封片胶制作硅藻永久性玻片. 做好的玻片在 Zeiss 显微镜下用 $10 \times 100$ 的油镜进行镜检,每 个玻片镜检的硅藻壳数量在 600 个左右 ${ }^{[19]}$. 硅藻种类的鉴定主要参考 Krammer 等的系统分类 书 $^{[6]}$ 及美国自 然科学研究院帕特里克环境研究中心提供的标准硅藻图谱.

\section{3 数据处理及统计分析}

每个样点中各种硅藻的数量用物种丰富度 $(S)$ 表示各硅藻物种的相对丰度即采样点样品中单种硅藻壳 体数占该样品检测到的所有硅藻壳体数量的百分比; 硅藻物种多样性用 Shannon-Wiener 指数 $\left(H^{\prime}\right)$ 表示; 群 落均匀度用 Pielou 均匀度指数 $(E)$ 表示; 用 Excel 对数据进行对数处理和做图. 除 $\mathrm{pH}$ 不做处理以外, 对其他 水化指标进行 $\lg (\mathrm{x}+1)$ 转换. 硅藻数据则选择至少在 2 个采样点出现, 且在一个样品中相对丰度 $>1 \%$ 的硅 藻物种用于分析. 经过上述处理后数据库中共有 10 个环境变量、53 个硅藻物种. 硅藻物种与环境因子之间 的关系在软件 CANOCO 4.5 中进行如下操作: 将物种数据进行除趋势对应分析 (DCA), 得到四个轴的最长 (最大值) 梯度长度 (Lengths of gradient) 小于 3(2.823), 因此本文选择线性模型中的圥余分析 (RDA) 进行 数据处理 ${ }^{[20]}$. 环境因子相关显著性由蒙特卡罗检验 (Monte Carlo permutation test) 测试, 并在 CANODRAW 4.5 中完成排序作图. 其他各种统计学检验则在 SPSS 18.0 中完成.

\section{2 结果与分析}

\section{1 环境因子}

5 个采样点 Chl. a 的浓度有相似的季节性变化规律 (表 1). Pearson 相关性分析表明,温度与 Chl. a 浓度 在 $\mathrm{S} 1 、 \mathrm{~S} 4$ 和 $\mathrm{S} 5$ 点的相关性较高, 其中在 $\mathrm{S} 4 、 \mathrm{~S} 5$ 点达到显著正相关 $(r=0.980 、 0.955, P<0.05)$, 而在 $\mathrm{S} 2 、 \mathrm{~S} 3$ 点则相关性不显著 $(r=0.563 、 0.345)$. 但在 $\mathrm{S} 3$ 点, 温度与 $\mathrm{PO}_{4}^{3-}-\mathrm{P}$ 则呈显著负相关 $(r=-0.987, P<0.05)$. $\mathrm{TN}$ 浓度在冬春季高于夏秋季, 最高值出现在 $\mathrm{S} 3$ 点的冬季 $(12.61 \mathrm{mg} / \mathrm{L})$, 最低浓度在 $\mathrm{S} 4$ 的秋季 $(0.78 \mathrm{mg} / \mathrm{L})$. 各样点理化因子之间相关性也不尽相同. 在 $\mathrm{S} 1$ 点, TP 和 TN 之间有极显著的正相关性 $(r=$ $0.992, P<0.01)$, 而 $\mathrm{NH}_{4}^{+}-\mathrm{N}$ 和 $\mathrm{NO}_{3}^{-}-\mathrm{N}$ 的相关系数为 $0.971(P<0.05)$, 但是在 $\mathrm{S} 4$ 点, $\mathrm{NH}_{4}^{+}-\mathrm{N}$ 则与 $\mathrm{TN}$ 呈显 著正相关 $(r=0.981, P<0.05)$, 而 $\mathrm{pH}$ 和 $\mathrm{NO}_{3}^{-}-\mathrm{N}$ 呈极显著负相关 $(r=-0.996, P<0.01)$. Chl. a 浓度可以用 来表示生物量, 本次实验中, 其最低值出现在 S5 点的冬季 $(0.47 \mu \mathrm{g} / \mathrm{L})$, 而在 S3 点的春季则达到最高值 $(40.4 \mu \mathrm{g} / \mathrm{L})$. 除了和温度有显著相关外, Chl. a 在 $\mathrm{S} 5$ 点还与 $\mathrm{PO}_{4}^{3-}-\mathrm{P}$ 呈显著负相关 $(r=-0.984, P<0.05)$.

表 12010 年东江惠州段不同季节的环境及水质特征

Tab. 1 Environmental characteristic and water quality in Huizhou section of Dongjiang River in four seasons of 2010

\begin{tabular}{cccccccccccc}
\hline 季节 & $\begin{array}{c}\mathrm{WT} / \\
{ }^{\circ} \mathrm{C}\end{array}$ & $\begin{array}{c}\mathrm{DO} / \\
(\mathrm{mg} / \mathrm{L})\end{array}$ & $\begin{array}{c}\mathrm{Cond} / \\
(\mu \mathrm{S} / \mathrm{cm})\end{array}$ & $\mathrm{pH}$ & $\begin{array}{c}\mathrm{SD} / \\
\mathrm{cm}\end{array}$ & $\begin{array}{c}\mathrm{PO}_{4}^{3-}-\mathrm{P} / \\
(\mathrm{mg} / \mathrm{L})\end{array}$ & $\begin{array}{c}\mathrm{TP} / \\
(\mathrm{mg} / \mathrm{L})\end{array}$ & $\begin{array}{c}\mathrm{NO}_{3}^{-}-\mathrm{N} / \\
(\mathrm{mg} / \mathrm{L})\end{array}$ & $\begin{array}{c}\mathrm{NH}_{4}^{+}-\mathrm{N} / \\
(\mathrm{mg} / \mathrm{L})\end{array}$ & $\begin{array}{c}\mathrm{TN} / \\
(\mathrm{mg} / \mathrm{L})\end{array}$ & $\begin{array}{c}\mathrm{Chl} . \mathrm{a} / \\
(\mu \mathrm{g} / \mathrm{L})\end{array}$ \\
\hline 春季 & 20.9 & 6.71 & 177.2 & 7.21 & 82 & 0.09 & 0.17 & 1.58 & 2.18 & 5.41 & 16.69 \\
夏季 & 30.7 & 6.40 & 141.1 & 7.61 & 62 & 0.02 & 0.08 & 0.53 & 1.38 & 2.96 & 15.15 \\
秋季 & 18.6 & 7.74 & 118.7 & 7.38 & 65 & 0.06 & 0.23 & 0.56 & 0.23 & 2.46 & 4.09 \\
冬季 & 14.1 & 7.74 & 198.4 & 7.24 & 87 & 0.13 & 0.19 & 1.20 & 2.40 & 6.96 & 2.71 \\
\hline
\end{tabular}




\section{2 硅藻种类与数量}

在东江惠州段 5 个采样点的 4 次采样 (共 20 个样品) 中,共鉴定出硅藻 40 属 153 种 (包括亚种和变 种). 20 个样品的物种丰富度指数在 23 60 之间 (表 2). S5 点冬季的各多样性指数均最高 (表 2); 和其它点 各季节的物种丰富度相比, S3 点各季节的物种丰富度最低. 由于 S4 点夏季的优势种的优势度增加, 相对丰 度较大 (图 2), 而该点物种多样性及均匀度最低(表 2)。

表 25 个采样点硅藻种群多样性指数的季节性变化

Tab. 2 Seasonal variations of $S, H^{\prime}$ and $E$ in diatom assemblages at the five sampling sites

\begin{tabular}{|c|c|c|c|c|c|c|c|c|c|c|c|c|c|c|c|}
\hline \multirow{2}{*}{ 季节 } & \multicolumn{3}{|c|}{ S1 } & \multicolumn{3}{|c|}{ S2 } & \multicolumn{3}{|c|}{ S3 } & \multicolumn{3}{|c|}{ S4 } & \multicolumn{3}{|c|}{ S5 } \\
\hline & $S$ & $H^{\prime}$ & $E$ & $S$ & $H^{\prime}$ & $E$ & $S$ & $H^{\prime}$ & $E$ & $S$ & $H^{\prime}$ & $E$ & $S$ & $H^{\prime}$ & $E$ \\
\hline 春季 & 34 & 3.03 & 0.86 & 26 & 3.32 & 1.02 & 30 & 3.45 & 1.02 & 38 & 3.35 & 0.92 & 58 & 4.72 & 1.16 \\
\hline 夏季 & 32 & 3.24 & 0.93 & 29 & 3.20 & 0.95 & 24 & 3.25 & 1.02 & 30 & 1.63 & 0.48 & 44 & 3.28 & 0.87 \\
\hline 秋季 & 37 & 3.83 & 1.06 & 43 & 3.77 & 1.00 & 30 & 3.12 & 0.92 & 38 & 4.02 & 1.11 & 49 & 4.41 & 1.13 \\
\hline 冬季 & 28 & 2.45 & 0.74 & 40 & 3.61 & 0.98 & 23 & 2.81 & 0.90 & 38 & 4.09 & 1.12 & 60 & 4.89 & 1.19 \\
\hline
\end{tabular}
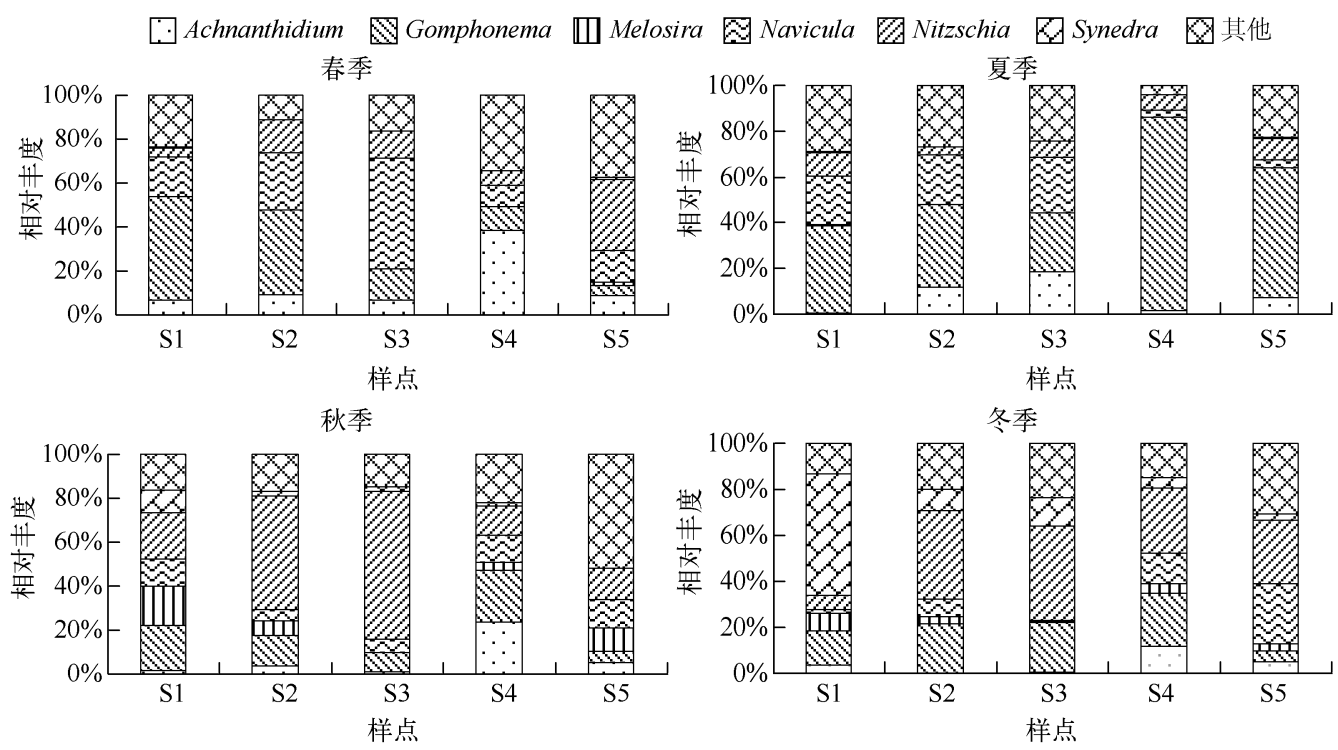

图 2 东江惠州段 5 个采样点不同季节优势属的相对丰度

Fig. 2 Relative abundance of dominant genera in each season at the five sampling sites

20 个样点中鉴定出主要的硅藻属有曲壳藻属 (Achnanthidium)、异极藻属 (Gomphonema) 、直链藻属 (Melosira)、舟形藻属 (Navicula)、菱形藻属 (Nitzschia)、脆杆藻属 (Synedra) 等. 但是不同的季节, 采样点的主 要种属所占百分比也有所不同 (图 2). 1 月份 (冬季) Synedra 和 Gomphonema 在各样点所占的比例较多, 其中 $\mathrm{S} 1$ 点优势种 S. ulna 所占的比例超过 50\%, Melosira varians 出现得较少; 在 S2、S3 点, N. palea 相对丰度分别 为 $30.6 \%$ 和 $34.8 \%$, 是主要优势种; 优势种 G. parvulum 在 S2、S3、S4 点的相对丰度都在 $20 \%$ 左右; 4 月份 (春季) 各点的 Gomphonema 和 Navicula 所占比例相对较高, 其中 S1 点的 G. parvulum 相对丰度达到 46.8\%, S3 点的 N. aff. subminuscula 的相对丰度为 $28.3 \%$; 另外, S4 点的 A. minutissima 相对丰度也超过 $37 \%$; S5 点 的优势种 N. palea 的相对丰度也有 $18 \%$. 和 4 月份一样, 8 月份 (夏季) 的优势属为 Gomphonema 和 Navicula, 其中 S1、S2、S4、S5 点的优势种均为 G. parvulum, 相对丰度都超过 30\%, 而 S4 点 Gomphonema 所占比例高达 $80 \%$; Navicula 属的优势种 N. subminuscula 在 S2、S3 点的相对丰度都超过 $20 \% ;$ S2、S3 点的 N. palea 在 11 月 (秋季) 的样品中又成为优势种, 在 $\mathrm{S} 3$ 点的相对丰度达 $39 \%, N$. intermedia 也达到 $18 \%$, 而 A. minutissima 
则成为 $\mathrm{S} 4$ 点的优势种.

\section{3 环境因子与硅藻种群的相互关系}

选取了 20 个样品中相对丰度都大于 $1 \%$, 并且在各样品中至少出现两次以上的硅藻物种 (共 53 个) 与 环境因子进行 RDA 分析, 结果表明第一轴的特征值为 0.307(图 3), 明显高于第二轴的特征值 0.095(表 3), 即藻属的分布主要受第一轴的环境因子的影响. 分析样点与环境变量的相关性可知,第一轴大致可以把采 样点从左到右分成春夏和秋冬的温度梯度变化. 蒙特卡罗检验的显著性分析结果表明, 水温在所有环境因 子中的贡献最大 $(S=0.19)$, 也达到显著水平 $(P=0.002)$. 另外, 溶解氧也较显著但贡献稍小 $(S=0.08$, $P=0.038)$. 轴一和轴二共同解释了 $40.2 \%$ 的硅藻组合的累积变率及 $60.9 \%$ 的物种与环境之间的累积百分 变量关系 (表 3 ).

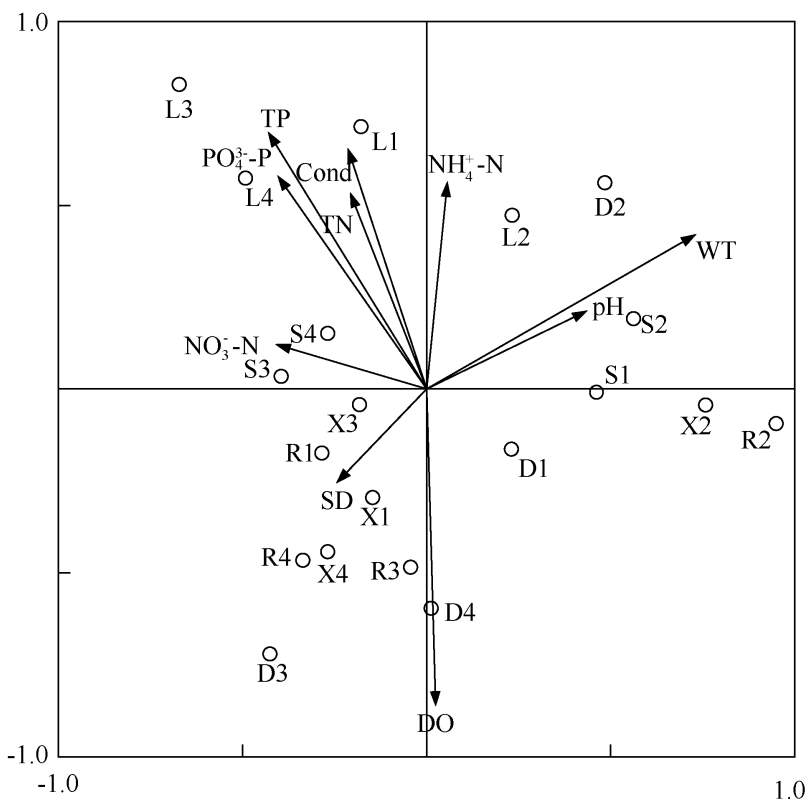

图 3 基于 RDA 方法的样点与环境变量的关系

(1一春季, 2一夏季, 3一秋季, 4一冬季, D- S1, W-S2, L-S3, X-S4, R- S5 )

Fig. 3 Results of RDA for environment variables and sampling sites

硅藻物种与环境变量 RDA 分析结果 (图 4 ) ,较好地反映了入选硅藻种类的分布与各 环境因子之间的相关性, 表明硅藻的种类分 布与水温、 $\mathrm{DO} 、 \mathrm{NH}_{4}^{+}-\mathrm{N} 、 \mathrm{PO}_{4}^{3-}-\mathrm{P} 、 \mathrm{pH}$ 等环境因 子都有一定的关系. Gomphonema exilissimum、 Mayamaea agrestis 、Navicula subminuscula、 Gomphonema parvulum 及 Gomphonema turris 等 藻种对温度具有指示作用, 其中 Gomphonema exilissimum、Gomphonema parvulum、Gomphonema turris 等 Gomphonema 属的种对水体的 $\mathrm{pH}$ 值有较好的指示作用; 而 Eunotia bilunaris、Nitzschla palea、Navicula glomus、Geissleria decussis 等藻类对磷则 有较好的指示性. 相对而言, 许多藻类都喜好在透明度较好、溶解氧较高及温度相对较低的春、秋季节的水 体环境中生长.

\section{表 $3 \mathrm{RDA}$ 主轴的基本特征}

Tab. 3 RDA axes summary statistics

\begin{tabular}{lcccc}
\hline & Axis1 & Axis2 & Axis3 & Axis4 \\
\hline 特征值 & 0.307 & 0.095 & 0.090 & 0.087 \\
物种与环境之间的相关性 & 0.885 & 0.865 & 0.840 & 0.813 \\
累积百分变量 & & & & \\
$\quad$ 物种之间 & 30.7 & 40.2 & 49.2 & 57.9 \\
$\quad$ 物种与环境之间 & 46.5 & 60.9 & 74.5 & 87.7 \\
\hline
\end{tabular}




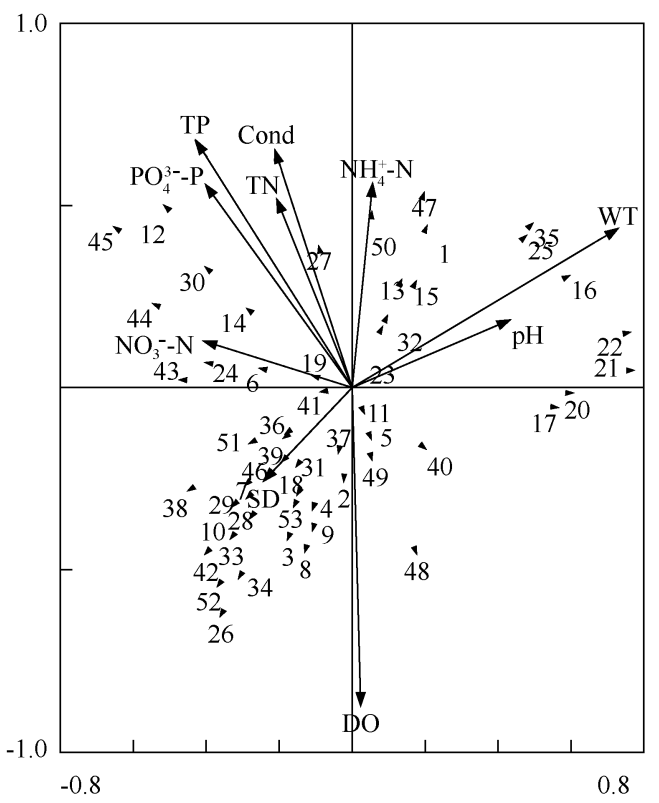

图 4 基于 RDA 方法的硅藻属种与环境变量的关系

(1-Achnanthidium exiguum,2-Achnanthidium minutissmum,3-Achnanthidium rivulare, $4-$ Adlafia minuscula,5-Amphora cf. strigosa,6-Amphora copulata,7-Bacillaria paradoxa,8-Cocconeis placentula,9-Cymbella tumida,10-Cymbella turigidula,11-Encyonema minuta,12-Eunotia bilunaris, 13-Eunotia incisa, 14-Geissleria decussis, 15-Gomphonema apuncto, 16-Gomphonema exilissimum,17 - Gomphonema insigne, 18 - Gomphonema kobayasii, 19-Gomphonema lagenula ,20-Gomphonema parvulius, 21 - Gomphonema parvulum, 22-Gomphonema turris, 23-Lemnicola hungarica, 24- Luticola goeppertiana, 25 - Mayamaea agrestis, 26-Melosira varians, 27 Navicula aff. subminuscula ,28 - Navicula canalis, 29 - Navicula evanida, 30 - Navicula glomus, 31 - Navicula hintzii,32 - Navicula minima,33 - Navicula parablis ,34 - Navicula rostellata ,35Navicula subminuscula,36- Navicula symmercia,37- Navicula viridula var. rostella,38 - Nitzschla angustata,39-Nitzschla clausii,40-Nitzschla filiformis,41-Nitzschla fossilis ,42-Nitzschla frustulum ,43-Nitzschla gracilis, 44-Nitzschla intermedia ,45-Nitzschla palea,46-Nitzschla subacicularis,47- Pinnularia subcapitata,48 - Planothidium frequentissmum,49-Planothidium lanceolatum,50-Sellaphora pupula,51 - Sellaphora seminulum,52-Surirella minuta,53-Synedra ulna)

Fig. 4 Results of RDA for environment variables and diatom assemblages

对 Gomphonema parvulum 和 Nitzschia palea 的研究发现, 在所有 20 个样品中这两个优势种的相对丰度都 大于 $1 \%$, 但其季节性变化明显. 如夏季 G. parvulum 在 S4 点的相对丰度达 $75 \%$ 以上, 在 S5 点也在 $45 \%$ 左 右, 但秋季其相对丰度在 S4、S5 点却只有 $15 \%$ 和不到 3\%（图 5), 而 $N$. palea 在 S2、S3 点的夏季相对丰度只 有 $14 \%$ 和 $12 \%$,而秋季相对丰度分别达到 $51 \%$ 和 $67 \%$.

\section{3 讨论}

由于人工底质具有面积可控性及附着藻类生长时间的可控性等特征 ${ }^{[10]}$, 自从 $1920 \mathrm{~s}$ 将人工底质用于附 着藻类的研究以来 ${ }^{[9]}$, 人工底质逐渐应用于河流及湖泊等水体的藻类生物监测中. 本次实验中, 通过放置人 工基质来附着着生硅藻, 研究着生硅藻群落的季节变化, 共鉴定出硅藻 40 属 153 种 (包括亚种和变种). 每 个采样点在不同季节的优势属基本相同, 但其相对丰度季节性变化却较大 (图 2). 如在 S1 点, 硅藻群落由 Gomphonema 属为主演变为在冬季以 Synedra 属为主; 在 S3 点, Navicula 属在春季为优势属,到夏季时有所降 低, 而秋季时则 Nitzchia 属占优势. 通过对各样点不同季节中的 G. parvulum 相对丰度分析( 图 5a), 发现 8 月 份该藻种在各点的相对丰度都较高, 其生长可能与 $\mathrm{pH}$ 值有关. 一般认为, 大多数异极藻属于碱性种, 生长在 
$\mathrm{pH} \geqslant 7$ 的水体中 ${ }^{[5]}$. 水体 $\mathrm{pH}$ 值, 尤其在偏碱性的水体中, 是影响硅藻群落结构变化的一个重要因子 ${ }^{[21]}$. 硅 藻群落对水体中 $\mathrm{pH}$ 值的变化非常敏感, 大部分硅藻物种生长所需的 $\mathrm{pH}$ 值范围都较窄, 如 A. minutissima 能 在 $\mathrm{pH}>5$ 的水体中生活, 但在 $\mathrm{pH}$ 低于 5 的水体里则很难存活; 也有少部分硅藻物种的 $\mathrm{pH}$ 值耐受性范围很 广, 如 Eunotia bilunaris 能在 $\mathrm{pH}$ 值范围为 $3 \sim 8$ 的环境中生长 ${ }^{[22]}$.

除 $\mathrm{pH}$ 外, 硅藻的生长还与营养状况有关, 如 G. parvulum 经常生长在污染较严重的水体中, 而 N. palea 等藻类是磷污染的指示种 ${ }^{[23]}$. 本次调查发现, N. palea 在 8 月份的相对丰度基本是每个点中最低的 (图 $5 b)$. 据 Besse-Lototskaya 等统计, 各种硅藻指数及大多数研究结果都表明, N. palea 是一种生活在富营养水 体中的藻类 ${ }^{[24]}$. S2、S3 两个采样点主要受西枝江支流的影响. 由于西枝江与东江交汇处的旁边是居民区, 受 人类活动影响较大, 污染比较严重, 营养盐浓度高于其它样点, 这两个样点 N. palea 的相对丰度一般也较 高; 但在 8 月份, 其相对丰度反而降低(图 5b), 而从全年的各季节来看, 除 $\mathrm{S} 1$ 点外, 其他 4 个点 $N$. palea 的 相对丰度在 8 月份也都是最低的(图 2), 可能是由于大雨冲刷所致. 惠州位于南亚热带地区, 雨季较长, 8 月 份正值丰水期,而 N. palea 是一种运动性较强的硅藻 ${ }^{[25]}$, 在水流较大的丰水期间, 其相对丰度很有可能因水 流的冲刷而被降低, 而 S1 点位于大坝处, 较其它样点的水流缓和, 因此相对丰度要高于其它样点. 除此以 外, N. palea 也有可能是受季节性温度变化的影响而导致其在夏季浓度低而秋冬季浓度相对较高. Moore 的 研究表明, 硅藻物种数量及分布会随着水温的改变而发生季节性变化 ${ }^{[26]}$, 硅藻较易生长在温度较低的春季 和秋季, 比如 Melosira varians 最适宜在温度为 $10 \sim 20^{\circ} \mathrm{C}$ 的环境中生长, 因此在春秋季节出现的频率和相对 丰度都较高 ${ }^{[5]}$, 与本文结果一致. 同时 RDA 分析也表明,研究区域内的河流中的大多数硅藻适合在春秋季 生长(图 4). 通过对两个优势种季节性变化的研究发现,秋冬季时期两种硅藻相对丰度变化较小,而夏秋时 期则变化较大 ( 图 5 ), 季节性变化趋势较明显.

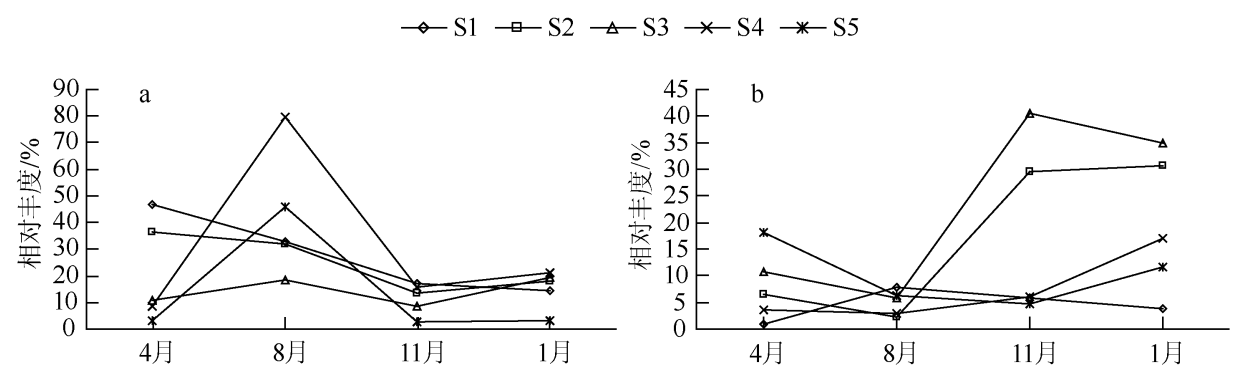

图 55 个采样点中优势种 G. parvulum (a) 和 $N$. palea $(\mathrm{b})$ 相对丰度的季节性变化

Fig. 5 Seasonal variations of the relative abundance of $G$. parvulum (a) and N. palea (b) at the five sampling sites

在河流中, 藻类和环境营养因子之间的关系比较复杂 ${ }^{[25]}$. 藻类生物量可以用来判断浮游和底栖藻类对 营养刺激的反应程度, 也可以用来评估某样点的底栖藻类生长速率 ${ }^{[27]}$, 因此常常用 Chl. a 的浓度来表示藻 类生物量的大小. 通常情况下, Chl. a 浓度越高, 藻类生物量越高. 相关性分析表明 Chl. a 浓度与温度呈显著 的正相关. 如在 5 个采样点中除 S3 点外 $(r=0.345)$, 其它几个点两者之间的相关性都较高, 其中在 S4、S5 点 达到显著水平 $(r=0.98,0.955 ; P<0.05)$. 春季是藻类大量生长繁殖的时期, 因此藻类生物量达到较高值, 但在秋冬季的生物量变化小于春夏季 (表 1). 温度变化不但影响藻类 Chl. a 浓度的大小, 还能影响藻类群落 组成,如 Soininen 等研究发现秋季后硅藻群落种群组成的稳定性较高,同样,他们认为这种现象主要与温度 的季节性变化有关 ${ }^{[28]}$. 本研究也认为春秋季 5 个采样点中的硅藻相对丰度要较夏冬季的高 (表 2).

与温度变化不同, $\mathrm{PO}_{4}^{3-}-\mathrm{P}$ 与 Chl. a 浓度呈负相关, 其中在 $\mathrm{S} 5$ 点达到显著水平 $(r=-0.984, P<0.05)$. 通常认为, 当河流中 $\mathrm{TN}: \mathrm{TP}>14$ 时, 磷则成为藻类生长的限制性因子, 此时水体中磷的含量决定水体的水质 状况及富营养化程度 ${ }^{[5]}$. Winter 等也发现底栖硅藻种群与总磷有显著相关性 ${ }^{[29]}$. 秋季不受磷因子影响的主 要原因可能是 8 月前期为丰水期, 雨量较大,水流较快, 从而降低了水体中的氮磷比 $(\mathrm{TN}: \mathrm{TP}<12)$; 而 $\mathrm{S} 1$ 点 位于大坝处, 水流相对较缓, 氮磷比相对较高 $(\mathrm{TN}$ : TP > 14), 因此磷此时成为藻类生长的限制性因子. 
S3 点在 5 个样点中水质状况最差, 藻种数在各季节都低于其它样点, 尤其在夏季, 其物种数在所有 20 个样品中最少 (表 2). 采样过程中发现该点所处的整个河流断面漂浮着大量的水葫芦, 导致水体中溶解氧明 显低于其它样点, 低溶解氧浓度抑制了硅藻的生长. 另外, 该点的电导率在 5 个点中最高. 电导率能反映水 体中各种离子的总浓度和总含量, 其大小与河流中溶解盐的含量有直接的关系 ${ }^{[5]}$. 电导率越高, 表明水体中 可溶性的离子越多. Potapova 等在研究美国一些河流底栖硅藻时发现, 电导率是影响硅藻种群分布的最重要 因子之一 ${ }^{[30]}$. RDA 双序图显示了电导率与 $\mathrm{WT} 、 \mathrm{NH}_{4}^{+}-\mathrm{N} 、 \mathrm{PO}_{4}^{3-}-\mathrm{P}$ 等其他环境因子对硅藻种群分布有类似的 影响(图 3), 蒙特卡罗检验的显著性分析结果表明, 电导率较重要, 但并不显著 $(S=0.09, P=0.22$ ). Leira 等也通过用 CCA 排序图研究发现, 除了电导率, 自然地理变量尤其是海拔和河流等级等因素也能够影响硅 藻群落结构 ${ }^{[31]}$.

\section{4 结论}

通过对东江惠州河段 4 个不同季节的附着硅藻群落调查,共鉴定出硅藻 40 属 153 种 (包括亚种和变 种). 主要的优势属有 Achnanthidium、Gomphonema、Melosira、Navicula、Nitzschia、Synedra 等,各藻属的相对丰度 具有明显的季节性变化, 在部分样点,一些硅藻种类的相对丰度甚至能达到 $50 \%$ 以上. 其中 Gomphonema parvulum 和 Nitzschia palea 为该河流段的广泛性藻种,根据种类季节性生长变化特征发现, G. parvulum 是一 个适碱性生长种. RDA 分析表明, 温度、营养因子、电导率、pH 值等能影响硅藻群落的分布和生长.

致谢: 感谢波特兰州立大学的 Yangdong Pan 教授在硅藻物种功能定类及部分参考文献方面给予的帮助!

\section{5 参考文献}

[ 1 ] Chathain BN, Harrington TJ. Benthic diatoms of the river deel: diversity and community structure. Biology and Environment, $2008,108(1)$ : 29-42.

[ 2 ] Kevern NR, Wilhm JL, van Dyne GM. Use of artificial substrata to estimate the productivity of periphyton. Limnology and Oceanography, 1966, 11(4) : 499-502.

[ 3 ] Stevenson RJ, Pan Y. Assessing ecological conditions in rivers and streams with diatoms. In: Stoermer EF, Smol JP eds. The diatoms: Applications to the environmental and earth sciences. Cambridge: Cambridge University Press, 1999: $11-40$.

[ 4 ] Barbour MT, Gerritsen J, Snyder BD et al. Rapid bioassessment protocols for use in streams and rivers: Periphyton, benthic macroinvertebrates, and fish. Washington, DC: United States Environmental Protection Agency, 1999.

[ 5 ] 何 琦. 增江流域和东江惠州段底栖硅藻多样性及分布特征 [学位论文]. 广州: 暨南大学, 2011.

[ 6 ] Krammer K, Lange-Bertalot H. Bacillariaceae. In: Pascher A, Ettl H, Gerloff J et al eds. Süsswasserflora von Mitteleuropa. Stuttgart: VEB Gustav Fisher Verlag, 1986-1991 : 3-874.

[ 7 ] Pienitz R, Smol JP, Birks HJB. Assessment of freshwater diatoms as quantitative indicators of past climatic change in the Yukon and Northwest Territories, Canada. Journal of Paleolimnology, 1995, 13(1) : 21-49.

[ 8 ] Rothfritz H, Juettner I, Suren AM et al. Epiphytic and epilithic diatom communities along environmental gradients in the Nepalese Himalaya: Implications for the assessment of biodiversity and water quality. Archiv Hydrobiol, 1997, 138(4) : 465-482.

[ 9 ] Cattaneo A, Amireault MC. How artificial are artificial substrata for periphyton? Journal of the North American Benthological Society, 1992, 11(2) : 244-256.

[10］裴国凤, 刘梅芳. 武汉东湖底栖藻类在不同基质上生长的比较. 湖泊科学, 2009, 21(3) : 357-362.

[11] Danilov RA, Ekelund NA. Comparison of usefulness of three types of artificial substrata when studying settlement patterns of periphyton in lakes of different trophic status. Journal of Microbiological Methods, 2001, 45(3) : 167-170.

[12] Tuchman M, Stevenson RJ. Comparison of clay tile, sterilized rock, and natural substrate diatom communities in a small stream in southeastern Michigan, USA. Hydrobiologia, 1980, 75(1) : 73-79.

[13］唐 涛, 蔡庆华,刘建康. 河流生态系统健康及其评价. 应用生态学报, 2002, 13(9) : 1191-1194.

[14] 董旭辉, 羊向东, 王 荣. 长江中下游地区湖泊富营养化的硅藻指示性属种. 中国环境科学, 2006, 26 (5): 570-574. 
[15] 何 琦, 韦桂峰, 刘 静等. 增江底栖硅藻种类多样性及分布特征. 热带亚热带植物学报, 2011, 19(3)： $245-253$

[16] Biggs BJF. Artificial substrate exposure times for periphyton biomass estimate in rivers. New Zealand Journal of Marine and Freshwater Research, 1988, 22(4) : 507-515.

[17] 林少君, 贺立静, 黄沛生等. 浮游植物中叶绿素 a 提取方法的比较与改进. 生态科学, 2005, 24(1): 9-11.

[18 ] Battarbee RW, Jones VJ, Flower RJ et al. Diatoms. In: Smol JP, Birks HJB, Last WM eds. Tracking environmental change using lake sediments, Vol 3: Terrestrial, algal, and siliceous indicators. Dordrecht: Kluwer Academic Publishers, 2001 : 155-202.

[19] Stevenson RJ, Pan Y, Van Dam H. Assessing environmental conditions in rivers and streams with diatoms. In: Smol JP, Stoermer EF eds. The Diatoms: Applications for the environmental and earth sciences, 2nd Edition. Cambridge: Cambridge University Press, 2010 : 57-85.

[20] 董旭辉, 羊向东, 刘恩峰等. 圥余分析 (RDA) 在简化湖泊沉积指标体系中的应用一一太白湖为例. 地理研究, $2007,26(3): 477-484$.

[21] 栾 卓, 范亚文, 门晓宇. 松花江哈尔滨段水域硅藻植物群落及其水质的初步评价. 湖泊科学, 2010, 22(1):8692.

[22] Van Dam H, Mertens A, Sinkeldam J. A coded checklist and ecological indicator values of freshwater diatoms from the Netherlands. Netherlands Journal of Aquatic Ecology, 1994, 28(1) : 117-133.

[23] Potapova M, Charles DF. Diatom metrics for monitoring eutrophication in rivers of the United States. Ecological Indicators, 2007, 7 (1) : 48-70.

[24] Besse-Lototskaya A, Verdonschot PFM, Coste M et al. Evaluation of European diatom trophic indices. Ecological Indicators, 2011, 11(2) : 456-467.

[25] Pan Y, Stevenson RJ, Hill BH et al. Spatial patterns and ecological determinants of benthic algal assemblages in Mid-Atlantic streams, USA. Journal of Phycology, 1999, 35(3) : 460-468.

[26] Moore JW. Seasonal succession of algae in a eutrophic stream in southern England. Hydrobiologia, 1977, 53 (2): 181-192.

[27] Smoot JC, Langworthy DE, Levy M et al. Periphyton growth on submerged artificial substrate as a predictor of phytoplankton response to nutrient enrichment. Journal of Microbiological Methods, 1998, 32 : 11-19.

[28] Soininen J, Eloranta P. Seasonal persistence and stability of diatom communities in rivers: are there habitat specific differences? European Journal of Phycology, 2004, 39(2) : 153-160.

[29] Winter JG, Duthie HC. Stream epilithic, epipelic and epiphytic diatoms: habitat fidelity and use in biomonitoring. Aquatic Ecology, 2000, 34: 345-353.

[30 ] Potapova M, Charles DF. Distribution of benthic diatoms in U. S. rivers in relation to conductivity and ionic composition. Freshwater Biology, 2003, 48(8) : 1311-1328.

[31] Leira M, Sabater S. Diatom assemblages distribution in catalan rivers, NE Spain, in relation to chemical and physiographical factors. Water Research, 2005, 39(1) : 73-82. 